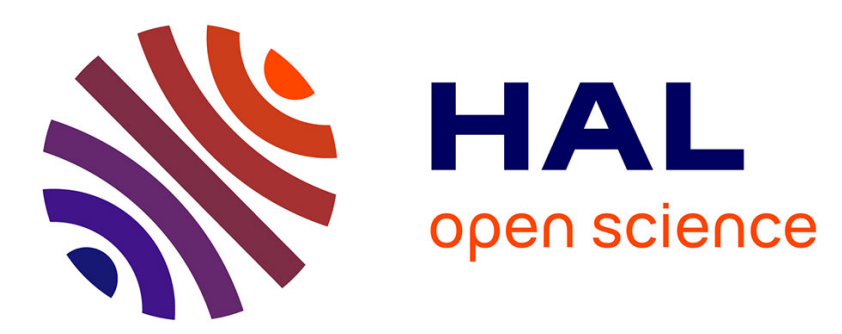

\title{
Détermination analytique de la résistance électrique de barreaux rectangulaires. Première partie: dispositifs à deux électrodes latérales
}

\author{
A. Morand, J. Robert
}

\section{- To cite this version:}

A. Morand, J. Robert. Détermination analytique de la résistance électrique de barreaux rectangulaires. Première partie: dispositifs à deux électrodes latérales. Revue de Physique Appliquée, 1981, 16 (3), pp.113-117. 10.1051/rphysap:01981001603011300 . jpa-00244900

HAL Id: jpa-00244900 https://hal.science/jpa-00244900

Submitted on 1 Jan 1981

HAL is a multi-disciplinary open access archive for the deposit and dissemination of scientific research documents, whether they are published or not. The documents may come from teaching and research institutions in France or abroad, or from public or private research centers.
L'archive ouverte pluridisciplinaire HAL, est destinée au dépôt et à la diffusion de documents scientifiques de niveau recherche, publiés ou non, émanant des établissements d'enseignement et de recherche français ou étrangers, des laboratoires publics ou privés. 


\title{
Détermination analytique de la résistance électrique de barreaux rectangulaires Première partie : dispositifs à deux électrodes latérales
}

\author{
A. Morand et J. Robert \\ Laboratoire de Génie Electrique des Universités Paris VI et Paris XI, E.S.E., Plateau-du-Moulon, 91190 Gif-sur-Yvette, France
}

(Reçu le 2 octobre 1980, révisé le 18 décembre 1980, accepté le 18 décembre 1980)

\begin{abstract}
Résumé. - La résistance électrique d'un barreau rectangulaire à deux électrodes latérales quelconques est calculée à l'aide de transformations conformes. La détermination de l'équation des équipotentielles et des lignes de courant est évoquée. Un abaque reliant les dimensions géométriques à la résistance électrique est établi pour des dispositifs symétriques. Dans le cas de barreaux rectangulaires longs ou infinis les grandeurs caractéristiques sont déterminées à l'aide d'expressions approchées. Différentes applications et généralisations des résultats obtenus sont envisagées.

Abstract. - The electric resistance of a rectangular bar with two lateral electrodes of any length or location, is obtained by means of conformal mappings. The determination of the equipotential and current lines equations is briefly described. A chart which relates the geometrical parameters to the electric resistance, is established for symmetrical configurations. For infinite or long rectangular bars, the characteristic quantities are calculated by means of approximate formulas. Several applications and generalizations are considered.
\end{abstract}

1. Introduction. - Cet article, consacré à la détermination de la résistance électrique de barreaux rectangulaires, est subdivisé en deux parties. La première est relative à des barreaux rectangulaires à deux électrodes. La seconde, qui porte sur des dispositifs à trois électrodes, sera publiée ultérieurement. L'étude effectuée repose sur l'emploi de transformations conformes. Elle exploite également les notions de plan complexe de référence et de géométries complémentaires qui sont exposées ci-dessous.

Soit un cylindre droit dont la directrice est une courbe plane, fermée, de forme quelconque. Deux sections droites définissent un disque plan, d'épaisseur uniforme $e$, constitué d'un matériau résistant homogène, de résistivité $\rho$ (Fig. 1). Deux portions de la surface latérale du disque sont métallisées afin de constituer les électrodes $\left(E_{1}\right)$ et $\left(E_{2}\right)$ reliées à des sources de potentiels constants $V_{1}$ et $V_{2}$. Dans ces conditions, l'étude des lignes de courant et des équipotentielles s'effectue dans le plan d'une section droite qui est identifié au plan complexe $(x, y)$ pour permettre l'emploi du potentiel complexe, $W$ [1]

$$
W(z)=U(x, y)+j V(x, y) .
$$

Dans cette expression $z$ est la variable complexe, $j$ le symbole imaginaire, $U$ et $V$ respectivement la fonction flux et le potentiel. Les lignes de courant, qui coïncident avec les lignes de force du champ électrique, sont définies par une valeur constante de la fonction $U$. Les valeurs $U_{1}$ et $U_{2}$, sur la figure 1 , repèrent les nappes superficielles de courant sur la face latérale du dispositif.

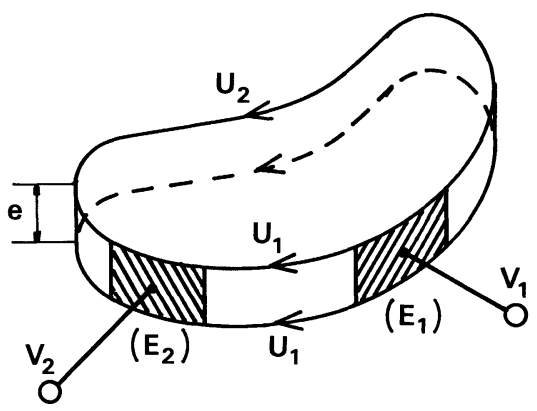

Fig. 1. - Disque cylindrique de matériau résistant homogène muni de deux électrodes métalliques latérales $\left(\mathrm{E}_{1}\right)$ et $\left(\mathrm{E}_{2}\right)$.

[Cylindrical disk of homogeneous resistive material. The curved side is fitted with two metallic electrodes $\left(E_{1}\right)$ and $\left(E_{2}\right)$.]

Soit d'autre part le plan complexe $(\xi, \eta)$, appelé plan de référence, pour lequel le potentiel complexe est tel que

$$
W(\zeta)=a \cdot \zeta=a(\xi+j \eta)
$$

Lorsqu'on choisit pour $a$ une valeur réelle, les équipotentielles et les lignes de champ sont des droites parallèles respectivement à l'axe des abscisses et à l'axe des ordonnées. Conformément au théorème de Riemann [2], il existe une transformation conforme (T) associant le domaine initial du plan $(x, y)$ à un rec- 
tangle du plan de référence $(\xi, \eta)$. L'existence de $(\mathrm{T})$ suffit pour la suite de l'étude sans que sa formulation explicite soit indispensable. Le rectangle de la figure $2 a$ est le transformé conforme d'une section droite du disque de la figure 1 . Les conditions aux limites sont les mêmes dans les deux cas. Ce rectangle est la section droite d'un barreau parallélépipédique rectangulaire présentant la même résistance électrique interélectrodes que le disque

$$
\mathcal{R}=\frac{\rho\left(V_{1}-V_{2}\right)}{e\left(U_{1}-U_{2}\right)}
$$

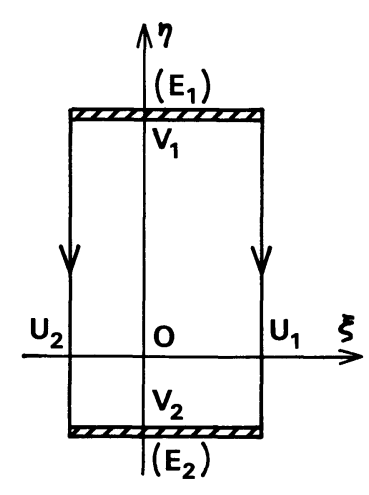

a)

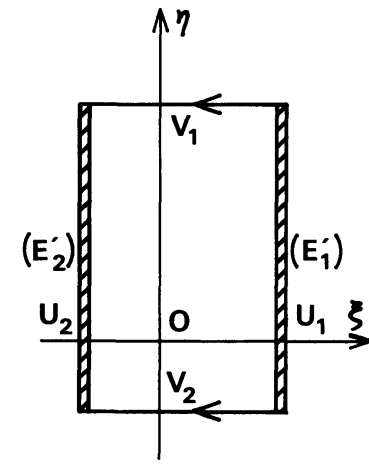

b)
Fig. 2. - Barreaux rectangulaires dans le plan de référence. a) Le rectangle représenté est le transformé conforme d'une section droite du disque de la figure 1.b) Dispositif complémentaire de celui du cas $a$. Il est obtenu en permutant les équipotentielles et les lignes de courant.

[Rectangular bars in the reference plane. a) A cross section through the disk of figure 1 is mapped onto the rectangle shown. In both cases, the boundary conditions and the reduced resistance are the same. $b$ ) Complementary device of $(a)$ resulting from the exchange of equipotential and current lines.]

Lorsque les variations de $V$ et de $U$ sont égales, c'est-à-dire lorsque le rectangle de la figure $2 a$ devient un carré, la résistance interélectrodes est la résistance par carré [3] relative au matériau considéré

$$
\mathcal{R}_{\square}=\frac{\rho}{e} .
$$

Afin d'obtenir des résultats généraux, l'étude porte sur des résistances réduites par rapport à la résistance par carré

$$
R=\frac{R}{\mathcal{R}_{\square}}=\frac{V_{1}-V_{2}}{U_{1}-U_{2}}=\frac{\Delta V}{\Delta U} .
$$

Cette relation permet d'évaluer la résistance électrique d'un disque de forme quelconque lorsque $V$ et $U$ ont des valeurs connues sur la frontière du domaine.

Pour la suite il est utile d'introduire la notion de dispositif complémentaire du dispositif étudié. Ce nouveau dispositif est obtenu en permutant le rôle des équipotentielles et des lignes de champ, c'est-à- dire en définissant le potentiel complexe du plan de référence par

$$
W(\zeta)=j a \zeta=-a \eta+j a \xi .
$$

Les électrodes $\left(E_{1}\right)$ et $\left(E_{2}\right)$ de la figure $2 a$ sont ainsi remplacées par les électrodes $\left(\mathrm{E}_{1}^{\prime}\right)$ et $\left(\mathrm{E}_{2}^{\prime}\right)$ de la figure $2 b$. Les valeurs aux limites des fonctions $V$ et $U$ sont les mêmes dans les deux cas. En pratique la géométrie complémentaire s'obtient en métallisant les intervalles entre les électrodes et en ôtant les électrodes du dispositif initial. Sa résistance, $R^{\prime}$, est telle que :

$$
R \cdot R^{\prime}=1 \text {. }
$$

Le présent article est consacré à des études de résistances mais il peut être noté que les résultats s'appliquent à de nouvelles déterminations, de capacités ou de résistances thermiques par exemple, sous réserve que les conditions aux limites soient conservées. Cette restriction est difficile à réaliser rigoureusement. En effet, pour une résistance électrique, le matériau du barreau canalise toutes les lignes de courant, alors que, dans le cas d'un condensateur, le matériau diélectrique du barreau ne canalise que partiellement les lignes de champ, ce qui entraîne l'apparition d'un flux de fuite. En supposant ce flux de fuite négligeable devant le flux principal, on peut vérifier que la capacité d'un dispositif du type $2 a$ est, en valeur réduite, égale à la résistance, réduite, du dispositif complémentaire $2 b$. Cette identité numérique se généralise à une localisation quelconque des deux électrodes.

2. Dispositifs rectangulaires à deux électrodes quelconques. - On considère maintenant le cas où le disque de la figure $1 \mathrm{a}$ une forme rectangulaire (Fig. 3a). Les extrémités, $M_{i}$, des électrodes métalliques $\left(E_{1}\right)$ et $\left(E_{2}\right)$ occupent des positions quelconques sur la frontière du rectangle. Les côtés du rectangle sont proportionnels aux nombres $K(n)$ et $K^{\prime}(n)$ qui sont des intégrales elliptiques complètes de première espèce liées à la valeur du paramètre $n$

$$
\begin{aligned}
K(n) & =\int_{0}^{\pi / 2} \frac{\mathrm{d} \theta}{\left(1-n \sin ^{2} \theta\right)^{1 / 2}} \\
K^{\prime}(n) & =K(1-n) .
\end{aligned}
$$

En pratique, la donnée des côtés du rectangle permet d'évaluer les nombres $n, K$ et $K^{\prime}$ à l'aide de tables de valeurs numériques [4], ou par le calcul grâce aux expressions qui les relient aux fonctions thêta [5]

avec

$$
n=\left[\frac{\theta_{2}(q)}{\theta_{3}(q)}\right]^{4} \quad K(n)=\frac{\pi}{2} \theta_{3}^{2}(q)
$$

$$
\begin{aligned}
\theta_{2}(q) & =\sum_{n=1}^{\infty}\left[2 q^{\left(n-\frac{1}{2}\right)^{2}}\right] \\
\theta_{3}(q) & =1+\sum_{n=1}^{\infty}\left[2 q^{n^{2}}\right] \\
q & =\exp \left[-\pi K^{\prime}(n) / K(n)\right] .
\end{aligned}
$$




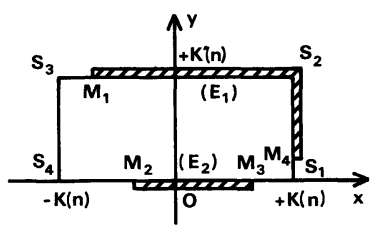

a)

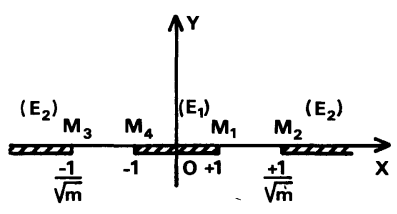

c)

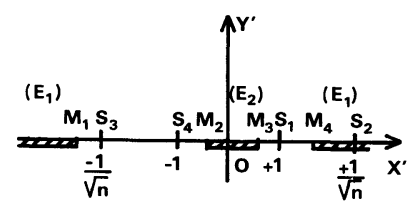

b)

d)

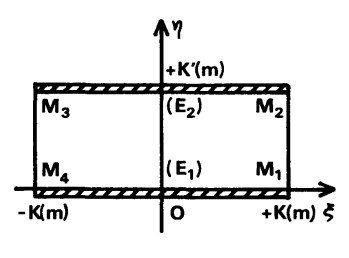

Fig. 3. - Transformations de domaines rectangulaires. a) Domaine rectangulaire initial caractérisé par le paramètre $n$. b) Demi-plan associé au rectangle (a) par une transformation de Schwarz-Christoffel. Les différents points se correspondent dans les deux cas mais la représentation est uniquement qualitative. c) Répartition d'électrodes symétrique, déduite du cas précédent par une transformation homographique. d) Domaine rectangulaire final caractérisé par le paramètre $m$.

[Transformations of rectangular bars. a) Initial rectangular system. The dimensions of the rectangle, and the location and size of $\left(E_{1}\right)$ and $\left(\mathrm{E}_{2}\right)$, are chosen at will. The parameter $n$ characterizes this system. $b$ ) Half plane related to $(a)$ by a Schwarz-Christoffel transformation. $c$ ) Symmetrical device deduced from $(b)$ by a bilinear transformation. d) Rectangle mapped on to (c) by a SchwarzChristoffel transformation. The double axial symmetry, with electrode lengths equal to the rectangle side results from the choice of the parameter $m$.]

Une transformation de Schwarz-Christoffel [6] associe l'intérieur du rectangle de la figure $3 a$ au demi-plan supérieur de la figure $3 b$. Cette transformation s'exprime à l'aide de la fonction elliptique de Jacobi sn, d'argument complexe $z$ et de paramètre $n$

$$
Z^{\prime}=\operatorname{sn}(z \mid n)
$$

(la définition usuelle de la fonction sn à l'aide du module $k$ a été écartée au profit de la relation précédente, où $n$ est le carré de $k$, pour des raisons de commodité de calculs).

Le demi-plan de la figure $3 b$ est soumis à une transformation homographique qui conduit au dispositif symétrique de la figure $3 c$. Entre les quatre coefficients de la transformation il n'est possible d'écrire que trois relations indépendantes [7]. Deux de ces relations traduisent la symétrie des points $M_{1}$ et $\mathbf{M}_{4}$, d'une part, et des points $\mathbf{M}_{2}$ et $\mathbf{M}_{3}$, d'autre part. La troisième est relative au facteur d'échelle. Dans le cas présent on choisit la valeur unité pour l'abscisse du point $\mathbf{M}_{1}$. Le domaine rectangulaire de la figure $3 d$ est obtenu par une nouvelle transformation de Schwarz-Christoffel qui fait intervenir le paramètre $m$ tel que, sur la figure $3 c$, l'abscisse de $\mathbf{M}_{2}$ soit égale à $m^{-1 / 2}$. Dans ces conditions, les électrodes $\left(E_{1}\right)$ et $\left(E_{2}\right)$ recouvrent complètement deux côtés opposés du rectangle et le plan de la figure coïncide avec le plan de référence. La détermination des nombres $K(m)$ et $K^{\prime}(m)$ fournit alors, à l'aide de la relation (1), la valeur de la résistance réduite du dispositif

$$
R=\frac{K^{\prime}(m)}{2 K(m)}
$$

Il peut être noté qu'en modifiant l'unité et le nombre $m$ sur la figure $3 c$, on aboutit, en $3 d$, à des rectangles présentant une seule symétrie ou une double symétrie axiale avec des longueurs d'électrodes différentes du côté du rectangle.

La transformation (T), qui associe les cas $a$ et $d$ de la figure 3 est égale au produit des deux transformations de Schwarz-Christoffel et de l'homographie qui viennent d'être définies. La formulation explicite de $(T)$ permet de déterminer le réseau d'équipotentielles et de lignes de courant pour la géométrie de la figure $3 a[8]$.

3. Dispositifs rectangulaires à deux électrodes symétriques. - Là suite de transformations représentées sur la figure 3 permet de calculer la résistance interélectrodes d'un rectangle quelconque. Cependant pour la majorité des applications pratiques les électrodes présentent différentes symétries. Lorsque la figure $3 a$ présente une symétrie par rapport à l'axe des ordonnées, l'homographie qui associe les figures $3 b$ et $3 c$ devient une homothétie. Il en résulte une simplification pour le calcul de la résistance. Afin de souligner le caractère pratique des résultats obtenus, ces calculs ont été utilisés pour tracer un abaque relatif à une géométrie présentant une double symétrie.

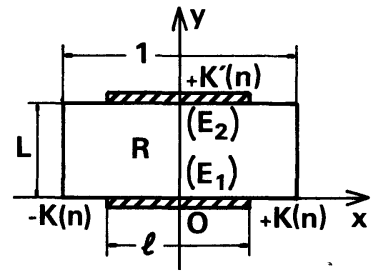

a)

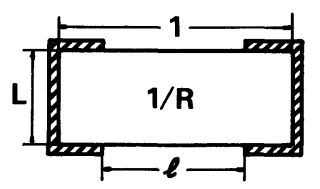

c)

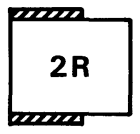

$\mathrm{R} / 2$

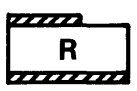

b)
Fig. 4. - Domaines correspondant à l'abaque de la figure 5. a) Rectangle présentant une double symétrie axiale. Les longueurs $l$ et $L$ sont des grandeurs réduites par rapport au côté horizontal pris pour unité. b) Dispositifs issus du cas initial par un fractionnement selon les axes de symétrie. $c$ ) Répartition complémentaire du cas $a$.

[Systems corresponding to the chart of figure 5.a) Rectangle with a double axial symmetry. The lengths $l$ and $L$ are dimensionless, the horizontal side being taken as unit length. b) System derived from (a) by cuts along the axes of symmetry. $c$ ) Complementary system of $(a)$. The reduced resistances of the two systems are reciprocal numbers.] 
axiale (Fig. 4a). Les électrodes ne recouvrent que partiellement deux côtés opposés du rectangle. Par fractionnement selon les axes de symétrie différents dispositifs (Fig. 4b) se déduisent du cas précédent. Leurs résistances respectives sont en relation simple avec la résistance de $(a)$ qui est évaluée à l'aide de l'abaque de la figure 5. Sur cet abaque les longueurs respectives $l$ des électrodes et $L$ du côté vertical, définies sur la figure $4 a$, sont réduites par rapport au côté horizontal pris comme unité. L'abaque fournit, par simple lecture, l'une des trois grandeurs $L, l$ ou $R$ lorsque les deux autres sont connues. L'emploi d'une gamme limitée de valeurs de $L$ sera justifié au paragraphe suivant. La résistance $R^{\prime}$ du dispositif complémentaire (Fig. $4 c$ ) est obtenue à l'aide de l'abaque et de la relation (2). Les dispositifs complémentaires de ceux de la figure $4 b$ sont obtenus par fractionnement du rectangle $4 c$, ce qui étend le champ d'applications de l'abaque. La figure 5 met en outre en évidence deux cas particuliers susceptibles de conduire à des applications. Tout d'abord, pour une longueur $L$ donnée, il apparaît que la résistance réduite $R$ varie peu lorsque la longueur d'électrode, $l$, est proche de l'unité. Ceci pourrait être mis à profit pour la réalisation de résistances de précision, par exemple au moyen d'un usinage permettant d'ajuster la valeur de $l$. A l'opposé, lorsque $l$ tend vers zéro, la résistance $R$

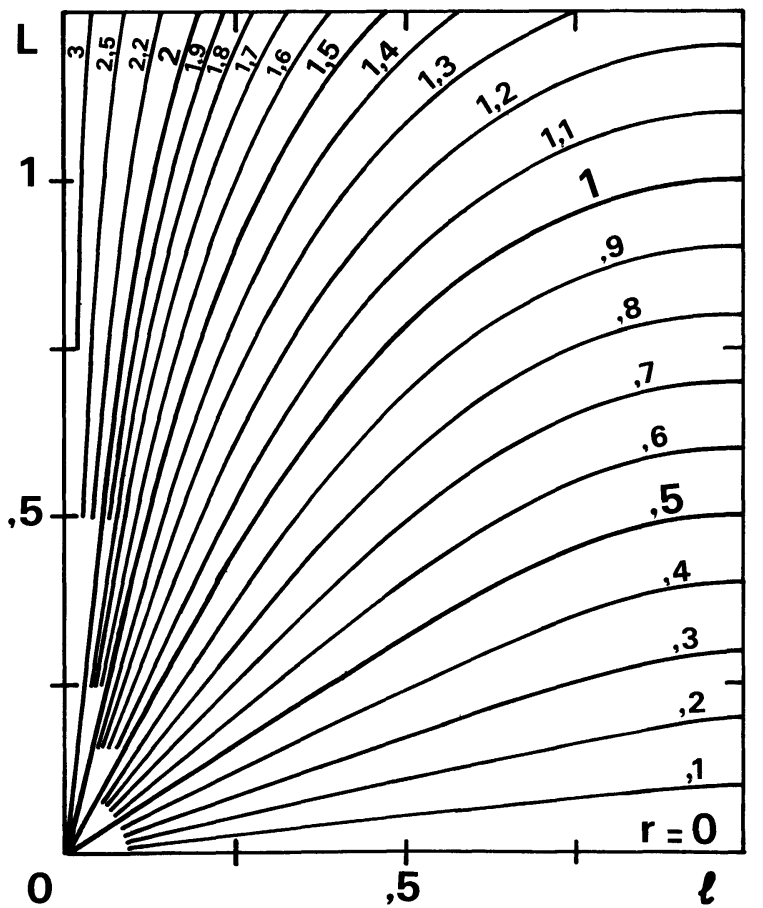

Fig. 5. - Abaque de résistances. Les notations sont celles de la figure $4 a$. L'abaque relie la longueur $L$ du côté du rectangle et la longueur $l$ des électrodes à la résistance réduite $R$ du dispositif. Pour les valeurs de $L$ ne figurant pas sur l'abaque les trois grandeurs sont reliées par l'expression (6).

[Resistance chart. The notations are the same as in figure $4 a$. The chart relates the rectangle side length $L$ and the electrode length $l$ to the reduced resistance $R$ of the device. For $L$ greater than unity, the relationship between the three quantities is expressed by equation (6).] varie dans des proportions importantes. Ceci pourrait être appliqué à la conversion en grandeur électrique d'un déplacement associé à $l$.

4. Barreaux longs. - Lorsque le rapport des côtés du rectangle diffère notablement de l'unité, des formules approchées peuvent être introduites dans les calculs de résistances. La figure $6 a$ représente le cas limite du rectangle de la figure $4 a$ lorsque le paramètre $n$ est égal à l'unité. Le nombre $K^{\prime}(n)$ est alors égal à $\pi / 2$ et $K(n)$ est infini. Pour ce barreau infini, la longueur $l$ des électrodes, réduite par rapport à la largeur du barreau prise pour unité, et la résistance $R$ sont reliées par l'expression approchée [9]

$$
l=\frac{1}{R}-\frac{2}{\pi} \ln 2 \text {. }
$$

L'erreur relative, sur $l$ ou $R$, résultant de l'emploi de cette formule, est inférieure à $1 \%$ lorsque $R$ est inférieur à un. Pour la géométrie complémentaire de la figure $6 b$, la relation (4), combinée avec la relation (2), conduit à

$$
l=R-\frac{2}{\pi} \ln 2
$$

Comme pour la figure 4 , le recours aux différentes symétries de la figure 6 étend le domaine d'application des relations (4) et (5) à de nouvelles géométries qui sont ici des barreaux semi-infinis ou infinis.

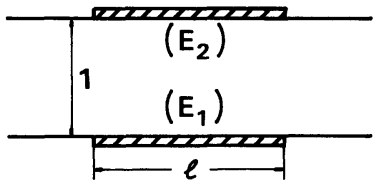

a)

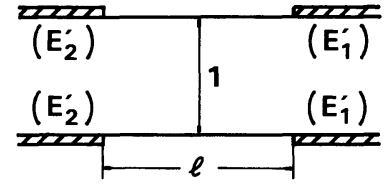

b)
Fig. 6. - Barreaux infinis. a) Barreau infini obtenu comme cas limite du rectangle de la figure $4 a$, lorsque le paramètre $n$ est égal à l'unité. $b$ ) Dispositif complémentaire du cas $a$.

[Infinite bars. a) Infinite bar as a limit for the rectangle of figure $4 a$ when the parameter $n$ equals unity. $b$ ) Complementary system of $(a)$.]

Pour des barreaux longs, les grandeurs caractéristiques peuvent également être reliées par des expressions approchées. La figure $7 a$ représente un barreau allongé dans la direction des ordonnées. Les électrodes symétriques ne recouvrent que partiellement les petits côtés du rectangle. Dans ce cas le paramètre $n$ tend vers zéro et le nombre $K(n)$ est voisin de $\pi / 2$. Les dimensions $L$ et $l$ sont réduites par rapport à la largeur du barreau prise pour unité. On désigne par $R_{0}$ la résistance du barreau pour lequel les électrodes recouvrent complètement les petits côtés du rectangle. Numériquement $R_{0}$ est 
égal à $L$. Avec ces notations, les grandeurs $l$ et $R$ sont reliées par l'expression [10]

$$
\sin \left(\frac{\pi l}{2}\right)=\exp \left[\frac{\pi}{2}\left(R_{0}-R\right)\right] .
$$

La validité de cette formule approchée a été déterminée en comparant les résultats auxquels elle conduit aux valeurs exactes obtenues par la méthode générale. On vérifie que la valeur de $l$ calculée à l'aide de la relation (6) entache $R$ d'une erreur relative inférieure à $1 \%$ lorsque la longueur $L$, égale à $R_{0}$, est supérieure à l'unité. Ceci justifie la gamme limitée de valeurs de $L$ prise en considération sur l'abaque de la figure 5 .

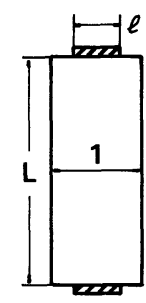

a)

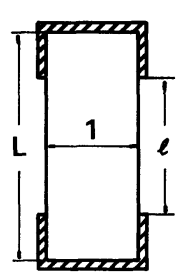

b)

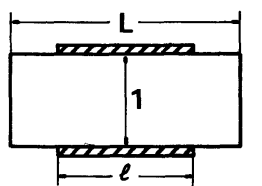

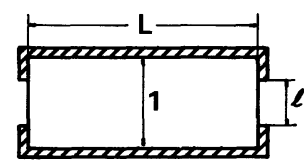

Fig. 7. - Barreaux longs. a) Barreau allongé déduit du rectangle de la figure $4 a$ en faisant tendre le paramètre $n$ vers zéro. $b$ ) Cas où les électrodes recouvrent toute la largeur et une fraction de la longueur du barreau. c) Dispositif complémentaire du cas $a$. d) Dispositif complémentaire du cas $b$.

[Long bars. a) Elongated bar derived from the rectangle of figure $4 a$ as the parameter $n$ tends to zero. $b$ ) Device with electrodes spreading over its whole width and $a$ part of its length. $c$ ) Complementary device of $(a)$. d) Complementary device of $(b)$.

Le barreau de la figure $7 b$, où les électrodes recouvrent les petits côtés et débordent sur les grands côtés du rectangle, constitue la transition entre les dispositifs des figures $4 c$ et $6 b$. Il peut être caractérisé par la relation (5). Le domaine de validité de l'approximité a été précisé à l'aide d'une étude numérique. Il apparaît que l'erreur relative sur la résistance est inférieure à $1 \%$ lorsque cette grandeur est supérieure à l'unité et la longueur $L$ supérieure à deux.

Les cas $c$ et $d$ de la figure 7 sont relatifs à un barreau allongé dans la direction des abscisses. Ces dispositifs, étant les compléments respectifs des figures $7 a$ et $7 b$, sont caractérisées par les mêmes expressions combinées avec la relation (2). Ainsi les grandeurs caractéristiques de la figure $7 c$ sont telles que

$$
\sin \left(\frac{\pi l}{2}\right)=\exp \left[\frac{\pi}{2}\left(\frac{1}{R_{0}}-\frac{1}{R}\right)\right]
$$

L'erreur relative sur $R$, due à l'emploi de cette relation, est inférieure à $1 \%$ lorsque la résistance $R_{0}$, égale numériquement à la longueur $L$, est supérieure à quatre. Pour la figure $7 d$, les grandeurs $l$ et $R$ sont liées par la relation (4) avec une erreur relative sur $R$ inférieure à $1 \%$ lorsque $R$ est inférieur à un et $L$ supérieur à quatre.

Pour les quatre barreaux de la figure 7 les symétries conduisent, comme sur la figure 4 , à de nouveaux dispositifs dont la résistance est en relation simple avec celle du cas initial.

5. Généralisation. - L'abaque de la figure 5 et les expressions (4) à (7) sont relatifs à des rectangles présentant une double symétrie. Le choix d'un facteur d'échelle différent pour la figure $3 c$ permet d'appliquer les résultats précédents à des rectangles ne présentant qu'une symétrie par rapport à un axe.

Un domaine circulaire peut être associé au domaine rectangulaire [11] afin d'évaluer la résistance de dispositifs circulaires à deux électrodes quelconques. Cette équivalence des deux types de domaines fournit en outre un moyen d'étude des rectangles à symétrie centrale. En effet, en associant une telle géométrie à un domaine circulaire, puis en effectuant une rotation de celui-ci, on aboutit à un rectangle possédant une symétrie axiale. Enfin, en ayant recours à des transformations conformes, les résultats précédents s'appliquent, par l'intermédiaire de la géométrie circulaire, à des domaines très divers.

6. Conclusion. - Cette étude conduit à la détermination de la résistance électrique de barreaux rectangulaires possédant deux électrodes de localisations et d'extensions quelconques. Le procédé mis en œuvre fournit l'équation des équipotentielles et des lignes de courant. Pour des rectangles présentant une double symétrie axiale, la relation entre les dimensions et la résistance du dispositif est traduite par un abaque, dans le cas général, et par des formules approchées, dans le cas particulier de barreaux longs ou infinis. Bien qu'il soit possible d'aboutir à des expressions rigoureuses, cette présentation souligne le caractère pratique des résultats obtenus.

Dans la seconde partie de cet article est abordée l'étude de barreaux rectangulaires à trois électrodes.

\section{Bibliographie}

[1] Durand, E., Electrostatique 2 (Masson, Paris) 1966, p. 238.

[2] WeBer, E., Electromagnetic theory (Dover, New York) 1965, p. 363 .

[3] Lyon-CAen, R., Circuits logiques intégrés (Masson, Paris) 1968 , p. 31

[4] Milne-Thomson, L. M., Jacobian elliptic function tables (Dover, New York) 1950, p. 106-109.

[5] Whittaker, E. T., Watson, G. N., Modern analysis (Cambridge University Press, Londres) 1963, p. 479.
[6] id. réf. 1, p. 273.

[7] id. réf. 1, p. 251.

[8] Morand, A., Thèse, Paris XI-Orsay (1980) p. 149-155.

[9] id. réf. 8, p. 165-167.

[10] id. réf. 8, p. 169.

[11] id. réf. 8, p. 287-294. 